\title{
The boundary action of the confining string and its universal contribution to the interquark potential
}

\section{Ferdinando Gliozzi*}

Dipartimento di Fisica, Università di Torino and

INFN, Sezione di Torino, Italy

E-mail: gliozzi@to.infn.it

\begin{abstract}
The request of Lorentz invariance of the effective string action describing the confining flux tube connecting a pair of static quarks implies that the first few terms in the expansion in the number of derivatives coincide with the Nambu-Goto action. The leading deviation from this action is a boundary term whose form is again determined by Lorentz invariance. Numerical simulations on $3 \mathrm{D} \mathbb{Z}_{2}$ gauge model allow to estimate and test this contribution.
\end{abstract}

Xth Quark Confinement and the Hadron Spectrum,

October 8-12, 2012

TUM Campus Garching, Munich, Germany

${ }^{*}$ Speaker. 


\section{The effective string picture of the confining flux tube}

The confining vacuum of gauge theories in the continuum limit is in a rough phase. This means in particular that long color flux tubes joining quark sources behave as string-like objects described in the far infrared [1] by two-dimensional massless free fields $X^{i}\left(\xi_{0}, \xi_{1}\right)(i=1, \ldots D-2)$. In a planar Wilson loop these describe the transverse displacements as functions of the world-sheet coordinates, labelled by $\xi_{\alpha}(\alpha=0,1)$. The $X^{i}$,s can also be seen as the Nambu-Goldstone modes of the spontaneously broken translation invariance in the transverse directions [2]. The IR limit of the string-like action is

$$
S_{0}=c_{0} \int d^{2} \xi \frac{1}{2}\left(\partial_{\alpha} X \cdot \partial^{\alpha} X+\ldots\right)
$$

where the ellipses stand for interaction terms. these are polynomials in the derivative of $X^{i}$ that will be specified below; they become important at intermediate distances. Early lattice calculations on fluid interfaces [3] suggested that the first correction to the Gaussian action was precisely the fourth-order term generated by the expansion of Nambu-Goto (NG) action in the number of derivatives. Note that $X^{i}$ 's do not have the canonical dimension of the free bosonic fields, owing to their significance of transverse displacements, then $c_{0}$ is a dimensionful physical constant that cannot be reabsorbed in a redefinition of $X^{i}$,s. It can be evaluated by measuring the mean square width of the flux tube as a function of the interquark distance [4]. In the NG action $c_{0}$ coincides with the string tension $\sigma$ and this has been confirmed in numerical evaluations of the string width in various abelian $[5,6]$ and non abelian $[7,8]$ gauge models. Numerical calculations of the energy spectrum of long closed flux tubes point increasingly to the NG string as the basic ingredient for describing the string-like degrees of freedom in confining gauge theories [9].

In order to understand the theoretical motivation of the special role of the NG action let us write the first few general terms of the string action allowed by the rotation and translation invariance $S O(2) \otimes I O(D-2)$ in the $\left(\xi_{0}, \xi_{1}\right)$ plane and in the $D-2$ transverse directions. In a planar Wilson loop encircling a planar surface of area $A$ we are left with the following expansion in the number of derivatives $[2,10]$

$$
\begin{aligned}
S\left[X^{i}\right] & =-\sigma A-c_{0} \int d^{2} \xi\left\{\frac{1}{2}\left(\partial_{\alpha} X \cdot \partial^{\alpha} X\right)\right. \text { [free string limit] } \\
& +c_{2}\left(\partial_{\alpha} X \cdot \partial^{\alpha} X\right)^{2}+c_{3}\left(\partial_{\alpha} X \cdot \partial_{\beta} X\right)\left(\partial^{\alpha} X \cdot \partial^{\beta} X\right) \text { [first non - Gaussian correction] } \\
& +c_{4}\left(\partial_{\alpha} X \cdot \partial^{\alpha} X\right)^{3}+c_{5}\left(\partial_{\alpha} X \cdot \partial_{\beta} X\right)^{2}\left(\partial_{\gamma} X \cdot \partial^{\gamma} X\right) \text { [second non - Gaussian corr.] } \\
& +c_{6}\left(\partial_{\alpha} \partial_{\beta} X \cdot \partial^{\alpha} \partial^{\beta} X\right)\left(\partial_{\gamma} X \cdot \partial^{\gamma} X\right) \text { [first term different from the NG - string] } \\
& \left.+O\left(\partial^{8} X^{4}\right)\right\}+ \text { boundary terms }+\ldots
\end{aligned}
$$

This action is written in the transverse or static gauge, where the only degrees of freedom are the physical ones. In 2004 Lüscher and Weisz [11] noted that comparison of the string partition function on a cylinder (Polyakov correlator) with the sum over closed string states in a Lorentz invariant theory yields strong constraints (open-closed string duality). They obtained, in particular,

$$
(D-2) c_{2}+c_{3}=\frac{D-4}{8},
$$

and $c_{0}=\sigma$. For $D=3$ there is only one fourth-order invariant and the above condition fixes uniquely the coefficient to be the one of the expansion of the NG action. 
As an aside, it is amusing to observe that if we assume that the $c_{i}$ 's do not depend on $D$ and use $D$ as a free parameter we get from (1.3)

$$
c_{2}+c_{3}=-\frac{1}{8} \text {. }
$$

The last equation was obtained in [12] by generalizing the Lüscher-Weisz argument to closed strings. Together the last two equations give

$$
c_{2}=\frac{1}{8} ; c_{3}=-\frac{1}{4}
$$

which coincide with the first coefficients of the expansion of the NG action in any space-time dimensions. It has been observed that an essential ingredient of the Lüscher-Weisz argument is the Lorentz invariance of the bulk space-time [13, 12]. It was subsequently argued [14] that the confining string action could be regarded as the effective action obtained from the underlying YangMills theory of the confining vacuum in presence of a large Wilson loop by integrating out all the massive degrees of freedom. This integration does not spoil the original Poincare invariance of the underlying gauge theory which is no longer manifest, but is realized through non-linear transformations of the string-like degrees of freedom encoded in the $X^{i}$ s. In particular the effective string action (1.2) should be invariant under the infinitesimal Lorentz transformation in the plane $(\alpha, j)$

$$
\delta X^{i}=-\varepsilon^{\alpha j} \delta^{i j} \xi_{\alpha}-\varepsilon^{\alpha j} X_{j} \partial_{\alpha} X^{i} .
$$

The recipe to write down Eq.(1.6) is very simple: the standard linear transformation which mixes coordinates and fields (see for instance figure 1) is followed by a reparametrization (the second term of (1.6)) by which the static gauge is restored. It is straightforward to verify that invariance

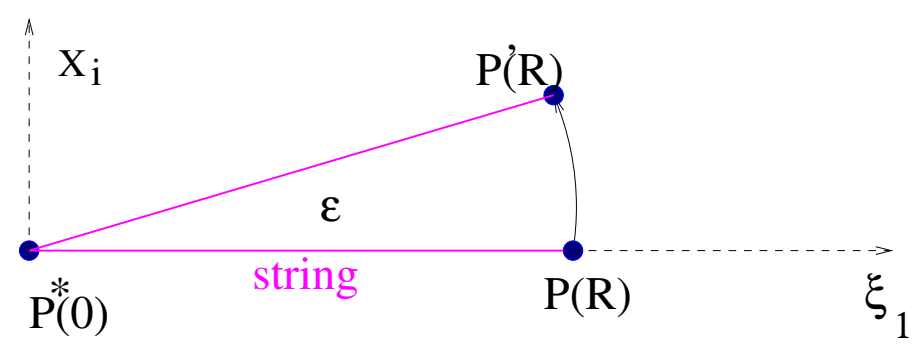

Figure 1: An infinitesimal rotation of a Polyakov loop correlator around the $\xi_{0}$ axis

under this transformation implies (1.5) and it is not difficult to argue that the whole NG action can be generated this way [14]. A general method that can be applied also in the construction of the effective action of more general extended object as the D-branes is to explicitly write and solve the recurrence relations induced by the request of invariance under (1.6) [15]. For instance, it easy to see that all the terms generated by $c_{2}$ and $c_{3}$ terms have the form

$$
I_{0}=\sum_{m=0}^{\infty} \sum_{n=1}^{\infty} c_{n, m}\left(\partial_{\alpha} X \cdot \partial^{\alpha} X\right)^{n}\left[\left(\partial_{\alpha} X \cdot \partial^{\beta} X\right)\left(\partial_{\beta} X \cdot \partial^{\alpha} X\right)\right]^{m},
$$

and the invariance under (1.6) implies [16, 17]

$$
(n+1) c_{n+1, m}+\left(\frac{n}{2}+m-\frac{1}{2}\right) c_{n, m}+(m+1) c_{n-1, m+1}=0,
$$


and

$$
(n+2) c_{n+2, m}+\left(m-\frac{1}{2}\right) c_{n+1, m}-(m+1) c_{n-1, m+1}=0 .
$$

The Lorentz transformation (1.6) acts only on terms with $m \geq 0$ and $n>0$. If we extend the recursion to include also the coefficient $c_{0,0}=-\sigma$, corresponding to the first term of (1.2), and solve these recurrence relations, we are left with $[16,17]$

$$
S_{N G}=-\sigma \sqrt{1+\left(\partial_{\alpha} X \cdot \partial^{\alpha} X\right)+\frac{1}{2}\left(\partial_{\alpha} X \cdot \partial^{\alpha} X\right)^{2}-\frac{1}{2}\left(\partial_{\alpha} X \cdot \partial^{\beta} X\right)\left(\partial_{\beta} X \cdot \partial^{\alpha} X\right)},
$$

which is precisely the NG action. If we had chosen a coefficient $c_{0,0} \neq-\sigma$ we had obtained the non-reparametrization invariant contribution $\left(\sigma+c_{0,0}\right) \int d^{2} \xi$.

At this point the question naturally arises: what is the first correction to NG action? It has been shown that the term associated with $c_{6}$ in (1.2) is actually absent, as it generates a total derivative $[16,17]$, and the first non vanishing correction has to be looked for in the boundary terms. Indeed quantum field theories on space-time manifolds with boundaries require, in general, the inclusion in the action of contributions localized at the boundary. The first non trivial term of this kind, proposed in [10], is, in the case in which the boundary is a Polyakov loop along the 0 axis,

$$
S_{1}=\frac{a}{4} \int d \xi_{0} \partial_{1} X \cdot \partial_{1} X
$$

where $a$ is a free parameter of dimension [length]; its contribution to the static potential is $-a \frac{(D-2) \pi}{24 r^{2}}$. It was subsequently observed that this term does not fulfill the requirements of the open-closed string duality [11]. From the point of view of the Lorentz symmetry, if we apply (1.6) to $S_{1}$ we get at once

$$
\delta S_{1}=-\frac{a}{4} \int \varepsilon^{1 i} d \xi_{0} \partial_{1} X_{i}+\text { higher order terms } \neq 0 .
$$

thus such a boundary term breaks explicitly the Lorentz invariance, hence it should be excluded from the boundary action.

The first boundary term compatible with Lorentz invariance is

$$
S_{\text {boundary }}=b \int d \xi_{0} \partial_{1} \partial_{0} X \cdot \partial_{1} \partial_{0} X+\ldots
$$

where now the free parameter $b$ has dimension $\left[(\text { length })^{3}\right]$. The requirement of Lorentz invariance generates an infinite sequence of terms of the form

$$
S_{\text {boundary }}=\int d \xi_{0} \sum_{k=0}\left[b_{k} \partial_{1} \partial_{0} X \cdot \partial_{1} \partial_{0} X\left(\partial_{1} X \cdot \partial_{1} X\right)^{k}+c_{k}\left(\partial_{1} \partial_{0} X \cdot \partial_{1} X\right)^{2}\left(\partial_{1} X \cdot \partial_{1} X\right)^{k}\right] .
$$

and the coefficients should obey the recurrence relations

$$
b_{n}+b_{n+1}=0,(n+1) c_{n}+n c_{n+1}=0, b_{n}+c_{n}+c_{n+1}=0
$$

whose solution gives

$$
S_{\text {boundary }}=b \int d \xi_{0}\left[\frac{\partial_{1} \partial_{0} X \cdot \partial_{1} \partial_{0} X}{1+\partial_{1} X \cdot \partial^{1} X}+\frac{\left(\partial_{1} \partial_{0} X \cdot \partial_{1} X\right)^{2}}{\left(1+\partial_{1} X \cdot \partial^{1} X\right)^{2}}\right]
$$


It is important to note that all terms appearing in (1.14) are characterized by the fact that the difference between the number of derivative and the number of fields is constant and equal to 2. This difference is called scaling and the expression (1.16) just constructed is the only Lorentz invariant of scaling 2. It is possible to show that there are no invariant of odd scaling [18]. It is easy to construct in this way boundary terms of higher scaling. For instance, there are two different Lorentz invariants of scaling four. One is simply the square of the boundary Lagrangian (1.16), the other is

$$
\begin{aligned}
\mathscr{L}_{4} & =\frac{\partial_{0}^{2} \partial_{1} X \cdot \partial_{0}^{2} \partial_{1} X}{1+\partial_{1} X \cdot \partial_{1} X}-\frac{\left(\partial_{0}^{2} \partial_{1} X \cdot \partial_{1} X\right)^{2}+4\left(\partial_{0}^{2} \partial_{1} X \cdot \partial_{0} \partial_{1} X\right)\left(\partial_{0} \partial_{1} X \cdot \partial_{1} X\right)}{\left(1+\partial_{1} X \cdot \partial_{1} X\right)^{2}} \\
& +4 \frac{\left(\partial_{0} \partial_{1} X \cdot \partial_{1} X\right)^{2}\left[\partial_{1} X \cdot \partial_{0}^{2} \partial_{1} X+\partial_{0} \partial_{1} X \cdot \partial_{0} \partial_{1} X\right]}{\left(1+\partial_{1} X \cdot \partial_{1} X\right)^{3}}-4 \frac{\left(\partial_{1} X \cdot \partial_{0} \partial_{1} X\right)^{4}}{\left(1+\partial_{1} X \cdot \partial_{1} X\right)^{4}} .
\end{aligned}
$$

It is also possible to write down the Lorentz invariants of higher scaling [18]. Of course the contribution of these boundary terms to the vacuum expectation value of Wilson loops or Polyakov correlators is too small to be of any practical utility in current numerical simulations.

\section{Quantum effects of the boundary action}

The boundary action (1.16) contributes to the Polyakov line correlator and to the Wilson loop through

$$
\left\langle S_{\text {boundary }}\right\rangle=\frac{1}{Z} \int \mathscr{D} X S_{\text {boundary }} \mathrm{e}^{S}
$$

where $Z$ is the partition function of the effective string. The lowest order contribution to the Polyakov line correlator (corresponding to the cylinder geometry of the world-sheet) is [14]

$$
\left\langle S_{\text {boundary }}\right\rangle_{\text {cylinder } L \times R}=-b \frac{\pi^{3} L}{60 R^{4}} E_{4}\left(\mathrm{i} \frac{L}{2 R}\right)+\text { higher order terms }
$$

where $E_{4}(\tau)$ is the second Eisenstein series, defined by

$$
E_{4}(\tau)=1-240 \sum_{n=1}^{\infty} \frac{n^{3} q^{n}}{1-q^{n}} ; \quad q=\mathrm{e}^{\mathrm{i} 2 \pi \tau}
$$

Similarly, the lowest order contribution to a rectangular Wilson loop $W(R, L)$ is [18]

$$
\left\langle S_{\text {boundary }}\right\rangle_{W}=-b \frac{\pi^{3}}{60}(L+R) \frac{1}{R^{4}} E_{4}\left(i \frac{L}{R}\right)+\text { higher order terms } .
$$

Notice that $\frac{1}{R^{4}} E_{4}\left(i \frac{L}{R}\right)=\frac{1}{L^{4}} E_{4}\left(i \frac{R}{L}\right)$, as required by the $R \leftrightarrow L$ symmetry.

This functional form has been checked in a set of simulations in the three-dimensional $\mathbb{Z}_{2}$ gauge model [18]. In particular, the evaluation of a set of Polyakov loop correlators in the low temperature regime and its comparison with eq. (2.2) supports the correctness of the effective string prediction and it is possible to extract a rather precise estimate of the coefficient $b$. To check the correct scaling behavior of $b$ this analysis has been performed for three different values of the bare coupling $\beta$. 


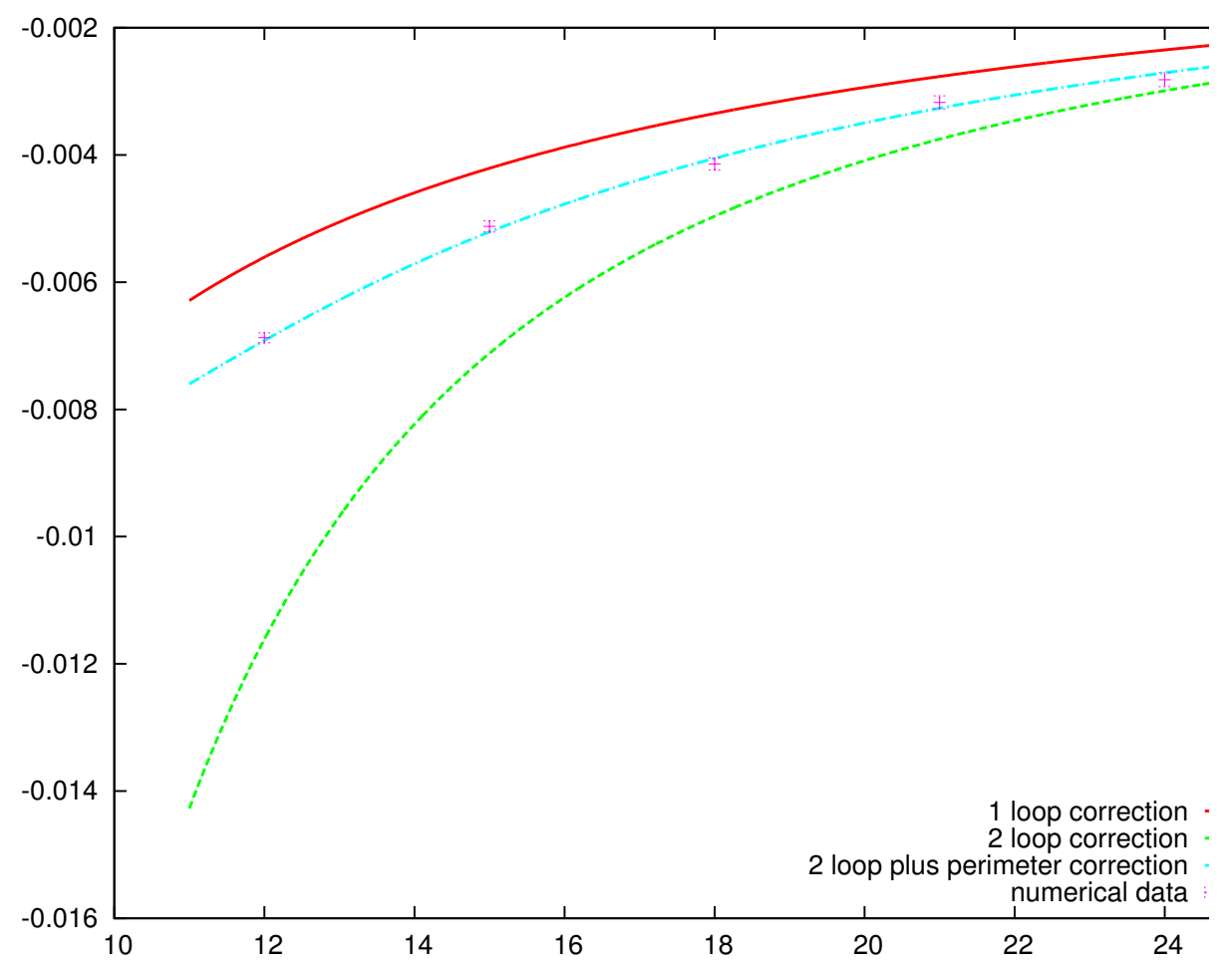

Figure 2: Wilson loop in $3 \mathrm{D} \mathbb{Z}_{2}$ gauge theory- Plot of $W(L, R) / W(L+1, R-1)$ with $R=\frac{4}{3} L$ at $\beta=$ 0.751805 .

The details of the simulation algorithm employed can be found in [19, 20]. As a basic update mechanism it has been used a multi-spin coded version of the standard Metropolis algorithm.

In order to eliminate the non-universal perimeter and constant terms from the expectation value of Polyakov loop correlators $P(R, L)$ (where $L$ is the length of the two loops and $R$ their distance) one typically measures the ratio:

$$
\mathscr{R}(R, L)=\frac{P(R+1, L)}{P(R, L)},
$$

which can be evaluated for large values of $R$ and $L$ with high precision.

The effective string form of this observable reads, up to the second order in $1 /(\sigma R L)$ expansion of the string action,

$$
\mathscr{R}(R, L)=\mathrm{e}^{-\sigma L} \frac{\eta\left(\mathrm{i} \frac{L}{2 R}\right)}{\eta\left(\mathrm{i} \frac{L}{2 R+2}\right)}\left(1+F_{2}(R+1, L)+F_{P}(R+1, L)-F_{2}(R, L)-F_{P}(R, L)\right),
$$

where $\eta$ is Dedekind's eta function, $F_{P}=\left\langle S_{\text {boundary }}\right\rangle$ is the leading correction coming from (2.2) and $F_{2}$ the standard two loop effective string contribution to the Polyakov loop correlator

$$
F_{2}(R, L)=-\frac{\pi^{2} L}{1156 R^{3} \sigma}\left[2 E_{4}\left(\mathrm{i} \frac{L}{2 R}\right)-E_{2}^{2}\left(\mathrm{i} \frac{L}{2 R}\right)\right]
$$


where the first Eisenstein series $E_{2}(\tau)$ is

$$
E_{2}(\tau)=1+24 \sum_{n=1}^{\infty} \frac{n q^{n}}{1-q^{n}}
$$

Using the fact that $\sigma$ is known with very high precision one can define a new observable which allows to isolate the boundary term:

$$
\mathscr{S}(R, L)=\mathrm{e}^{\sigma L} \frac{\eta\left(\mathrm{i} \frac{L}{2 R+2}\right)}{\eta\left(\mathrm{i} \frac{L}{2 R}\right)} \mathscr{R}(R, L)-F_{2}(R+1, L)+F_{2}(R, L) .
$$

When $L \gg 2 R$ one has

$$
\mathscr{S}(R, L) \simeq-b \frac{L \pi^{3}}{15 R^{5}} .
$$

A remarkable consistency check of these numerical evaluations is that the adimensional expression $b \sqrt{\sigma}^{3}$ is almost constant. This quantity can be used to define the continuum limit value of $b$, which can thus be considered as a new physical scale of the model, on the same ground as the string tension $\sigma$ or the glueball mass $m_{g}$. The estimate of [18] was $b \sqrt{\sigma}^{3} \sim 0.032(2)$. It is interesting to notice that this value in the $3 d \mathbb{Z}_{2}$ gauge model is very similar (but ten times more precise) to the same parameter measured in the $3 d S U(2)$ gauge model in [21].

Using the above value of $b$ it has been also tested [18] the leading boundary contribution to the Wilson loop given by eq. (2.4). Since one knows $\sigma$ and $b$ from independent simulations on Polyakov correlators, there are no free parameters left.

As an example, in figure 2 we report some numerical data for the ratio

$$
\mathscr{R}(L, R)=\frac{W(L, R)}{W(L+1, R-1)} .
$$

The agreement between the data and the effective string prediction is impressive and, within the precision of the data, the boundary correction is needed to correctly describe them.

\section{References}

[1] M. Luscher, K. Symanzik, and P. Weisz, Anomalies of the Free Loop Wave Equation in the WKB Approximation, Nucl.Phys. B173 (1980) 365.

[2] M. Luscher, Symmetry Breaking Aspects of the Roughening Transition in Gauge Theories, Nucl.Phys. B180 (1981) 317.

[3] M. Caselle, R. Fiore, F. Gliozzi, M. Hasenbusch, K. Pinn, et al., Rough interfaces beyond the Gaussian approximation, Nucl.Phys. B432 (1994) 590-620, arXiv: hep-lat/ 9407002 [hep-lat].

[4] M. Luscher, G. Munster, and P. Weisz, How Thick Are Chromoelectric Flux Tubes?, Nucl.Phys. B180 (1981) 1.

[5] M. Caselle, F. Gliozzi, U. Magnea, and S. Vinti, Width of long color flux tubes in lattice gauge systems, Nucl.Phys. B460 (1996) 397-412, arXiv: hep-lat/9510019 [hep-lat] .

[6] P. Giudice, F. Gliozzi and S. Lottini, Quantum broadening of $k$-strings in gauge theories, JHEP 0701 (2007) 084 [hep-th/0612131]. 
[7] F. Gliozzi, M. Pepe, and U.-J. Wiese, The Width of the Confining String in Yang-Mills Theory, Phys.Rev.Lett. 104 (2010) 232001, arXiv:1002.4888 [hep-lat].

[8] F. Gliozzi, M. Pepe, and U.-J. Wiese, The Width of the Color Flux Tube at 2-Loop Order, JHEP 1011 (2010) 053, arXiv:1006.2252 [hep-lat].

[9] M. Teper, Large $N$ and confining flux tubes as strings - a view from the lattice, Acta Phys. Polon. B40 (2009) 3249-3320, arXiv:0912.3339 [hep-lat].

[10] M. Luscher and P. Weisz, Quark confinement and the bosonic string, JHEP 0207 (2002) 049, arXiv:hep-lat/0207003 [hep-lat].

[11] M. Luscher and P. Weisz, String excitation energies in $S U(N)$ gauge theories beyond the free-string approximation, JHEP 07 (2004) 014, arXiv : hep-th / 0406205.

[12] O. Aharony and E. Karzbrun, On the effective action of confining strings, JHEP 06 (2009) 012, arXiv:0903.1927 [hep-th].

[13] H. B. Meyer, Poincare invariance in effective string theories, JHEP 05 (2006) 066, arXiv:hep-th/0602281.

[14] O. Aharony and M. Field, On the effective theory of long open strings, JHEP 1101 (2011) 065, arXiv:1008.2636 [hep-th].

[15] F. Gliozzi, Dirac-Born-Infeld action from spontaneous breakdown of Lorentz symmetry in brane-world scenarios, Phys.Rev. D84 (2011) 027702, arXiv:1103.5377 [hep-th] .

[16] F. Gliozzi and M. Meineri, Lorentz completion of effective string (and p-brane) action, JHEP 1208 (2012) 056 [arXiv:1207.2912 [hep-th]].

[17] M. Meineri, Lorentz completion of effective string action, in proceedings of Xth Quark Confinement and the Hadron Spectrum.

[18] M. Billo, M. Caselle, F. Gliozzi, M. Meineri, and R. Pellegrini, The Lorentz-invariant boundary action of the confining string and its universal contribution to the inter-quark potential, JHEP 05 (2012) 130, arXiv:1202.1984 [hep-th].

[19] M. Caselle, M. Hasenbusch, and M. Panero, String effects in the 3-d gauge Ising model, JHEP 0301 (2003) 057, arXiv:hep-lat/0211012 [hep-lat].

[20] M. Billo, M. Caselle, and R. Pellegrini, New numerical results and novel effective string predictions for Wilson loops, JHEP 1201 (2011) 104, arXiv:1107.4356 [hep-th] .

[21] B. B. Brandt, Probing boundary-corrections to Nambu-Goto open string energy levels in 3d SU(2) gauge theory, JHEP 02 (2011) 040, arXiv: 1010.3625 [hep-lat ] . 